\title{
Mitochondria Inspired Nanoparticles with Microenvironment-Adapting Capacities for On-Demand Drug Delivery after Ischemic Injury
}

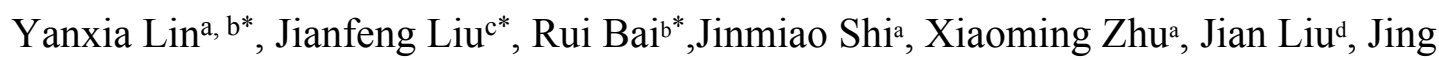
Guo $^{\mathrm{b}}$, Wei Zhang ${ }^{\mathrm{e}}$, Huiliang Liu ${ }^{\mathrm{b} \#}$, Zhiqiang Liu ${ }^{\mathrm{a} \#}$

a. Institute of Military Cognition and Brain Sciences, Academy of Military Medical Sciences, Beijing 100850, China

b. Department of Cardiology, the Third Medical Center of PLA General Hospital, Beijing, 100039, China.

c. Department of Cardiology, the First Medical Center of PLA General Hospital, Beijing, 100853, China.

d. Department of Nuclear Medicine, the First Medical Center of PLA General Hospital, Beijing, 100853, China.

e. Department of Orthopaedics, Chinese PLA General Hospital, No.28 Fuxing Road, Beijing, 100850, PR China.

* The firstly three authors contributed equally to this work.

\#Correspondance to Wei Zhang (bszw@hotmail.com), Huiliang Liu (1h1518@vip.sina.com) and Zhiqiang Liu (zhiqiangliu_amms@163.com, leading correspondence) 


\section{Supplementary Methods/Experimental}

Construction of oxygen-sensing circular DNA system. Oxygen-sensing DNA system was constructed from the plasmid pGL4.73[hRluc/SV40] (pGL4.73/Rluc), which was purchased from Promega. The sequence of EPO enhancer was searched in NCBI as shown in Table S1. The double-tandem EPO enhancers were chemically synthesized and inserted into vector to obtain a pEZclone-NRS-Amp-Blunt-HC-EPO(pEPO) plasmid (restriction enzyme Xho I and $\mathrm{Bgl}$ II sites were added at the upstream and downstream of EPO enhancers respectively). Then, the double-tandem EPO enhancer fragments were amplified using polymerase chain reaction (PCR). VEGF165 gene was amplified from pcDNA3 .1 plasmids (Kindly provided from Professor Hong Jiang). The forward and reverse primers were added with restriction enzyme HindIII and Xba I sites, respectively. pGL4.73 plasmid and double-tandem EPO enhancers were digested with Xho I and Bgl II (Promega). After purification using DNA purification kit (Tiangen), the digested pGL4.73 and EPO enhancers were linked with T4 DNA ligase to obtain circular pGL4.73/EPO/Rluc DNA. The linked DNA was transformed into Escherichia coli. The transformed bacterial cells were smeared onto LB solid medium supplemented with ampicillin. The single clone was selected and transferred into LB liquid medium (+Ampicillin) for amplification. After sequencing, clones with right DNA sequences were stored for next usage. VEGF165 gene was inserted into pMD18-T and transformed into Escherichia coli for amplification. As described above, the sequenced clone with right DNA sequence was selected. pMD18-T vectors carrying VEGF165 were digested with restriction enzyme HindIII and Xba I . After electrophoresis, the VEGF165 fragments were recovered and purified. The above pGL4.73/EPO/Rluc DNA was digested by restriction enzymes 
HindIII and Xba I to remove Rluc. Then, the resulted DNA products were linked with VEGF165 fragment to obtain circular pGL4.73/EPO/VEGF DNA. With the same method, pGL4.73/Rluc DNA was digested by the two restriction enzymes and linked with VEGF165 fragments to obtain pGL4.73/VEGF DNA. The successful insertion of VEGF165 gene was detected by PCR, and further verified by HindIII and Xba I cutting to obtain the target fragments. The above plasmids were transformed into 293T cells with Entranster-H4000 kit (Engreen Biosystem Co, Ltd.). The Rluc activities were detected via bioluminescent signals and VEGF expression was detected by reverse-transcription PCR. The hypoxic condition was simulated with $\mathrm{CoCl} 2$ treatment $(100 \mu \mathrm{M})$ and hypoxic incubator, respectively.

Cell culture. 293T cells were preserved in our lab. Dulbecco's Modified Eagle Medium (Gibco) supplemented with 10\% fetal bovine serum (FBS, Gibco) was used for cell growth and expansion. For long-term observation under changing microenvironments (hypoxia and normaxia), 4\% FBS was used to slow cell proliferation. Cardiac cells were isolated from neonatal Sprague Dawley rats according to the previous report ${ }^{1}$.

Bioluminecent imaging. Bioluminescent imaging was performed using a highly sensitive charge-coupled device (CCD) camera (IVIS50, Xenogen, USA). Ranilla Luciferase detection kit (Promega) was used according to the manufacturer's instruction.

Intracellular reactive oxygen species(ROS) detection. Reactive oxygen species assay kit was purchased from Beyotime Biotechnology Inc. The kit was used according to the manufacturers' instruction. Briefly, DCFH-DA probe was diluted with serum-free medium at the ratio of 1:1000. Then, the DCFH-DA solution was added to cultured cells and incubated for $20 \mathrm{~min}$ at $37^{\circ} \mathrm{C}, 5 \% \mathrm{CO}_{2}$ incubator. The DCFH-DA solution was removed and the cells 
were washed with serum-free medium for 3 times. Hoechst33342 was used for nucleus staining. The cells were observed under fluorescent microscope (Olympus). The ratio and intensity of DCFH-DA staining were quantified with Image Pro software.

CCK8 assay and TUNEL staining. Cell viabilities and apoptosis were evaluated by CCK8 (Cell Counting Kit-8) assay and TUNEL staining according to manufacturers' instructions. CCK8 assay kit and TUNEL staining kit were purchased from Dojindo and Beyotime Biotechnology Inc, respectively.

PET/CT imaging. At one week after surgery, $400 \mu \mathrm{CI}{ }^{18} \mathrm{~F}-\mathrm{FDG}$ was injected into each mouse through tail vein. After $40 \mathrm{~min}$ metabolism, mice were anesthetized by inhalation of $2 \%$ isoflurane. A Super Nova PET/CT ( SNPC-103 ) instrument (PINGSENG Healthcare Inc) was used for PET/CT imaging according to the previous report. ${ }^{2}$

Echocardiogram. At 4 week, mice were anesthetized with 2\% isoflurane in $100 \%$ oxygen. The cardiac function was evaluated with echocardiogram according to the previous report. ${ }^{3}$ Briefly, the chest area of mice was shaved and ultrasound coupling gel was applied to the area before measurement. A Vevo 1100 Imaging System (VisualSonics Inc, Toronto, ON, Canada) was used to acquire M-mode echocardiographic images in parasternal short and long axis views. Then, LV functional parameters were calculated, including Left-ventricular end-diastolic diameter (LVEDD), left-ventricular end-systolic diameter (LVESD), left ventricular shortening fraction (LVFS \%) and left ventricular ejection fraction (LVEF \%).

Histological and immunohistochemical analysis. Mice were sacrificed by intraperitoneal injection of over-dose pentobarbital sodium. Hearts were explanted and harvested. After fixation in 4\% paraformaldehyde, hearts were dehydrated with gradient concentration alcohol 
and embedded into paraffin. Then, $4 \mu \mathrm{m}$ thick sections were prepared for histological and immunohistological staining. For infarct size and fibrosis evaluation, Masson trichrome staining was performed and the total sections were scanned to acquire the whole images of heart horizontal planes. The infarct size was calculated as follows: Inner infarct size was calculated as infarct inner circumference/ LV circumference; outer infarct size was calculated as infarct outer circumference/the whole circumference of heart horizontal plane. The fibrosis was calculated as fibrotic area/total area in images. The fibrotic area was identified using Image Pro software. For vascularization assay, heart sections were immunostained with anti-CD34 antibodies (Boster, Wuhan, China). The positive stained areas were identified using Image Pro software. The vessel densities were calculated as the number of vessels per field under microscope.

Statistical analysis. Quantified data were expressed as mean \pm standard deviation. Statistical analysis was performed with GraphPad Prism 7 software. A Student's t-test was employed for two-group comparison while one-way ANOVA with Tukey's posthoc test was employed for three or more group comparison. A value of $p<0.05$ was considered as statistically significant. 
Table S1 Sequencing of inserted tandem EPO enhancers

\begin{tabular}{ll}
\hline Name & Base Sequence \\
\hline \multirow{2}{*}{ Primary } & CTCGAGGCCCTACGTGCTGTCTCACACAGCCTGTCTGACCTCTCGACCTACC \\
& GGCGGCCCTACGTGCTGTCTCACACAGCCTGTCTGACCTCTCGACCTACCG \\
GCGAGATCT & CTCGAGGCCCTACGTGCTGTCTCACACAGCCTGTCTGACCTCTCGACCTACC \\
& GGCGGCCCTACGTGCTGTCTCACACAGCCTGTCTGACCTCTCGACCTACCG \\
& GCGAGATCT \\
\hline
\end{tabular}




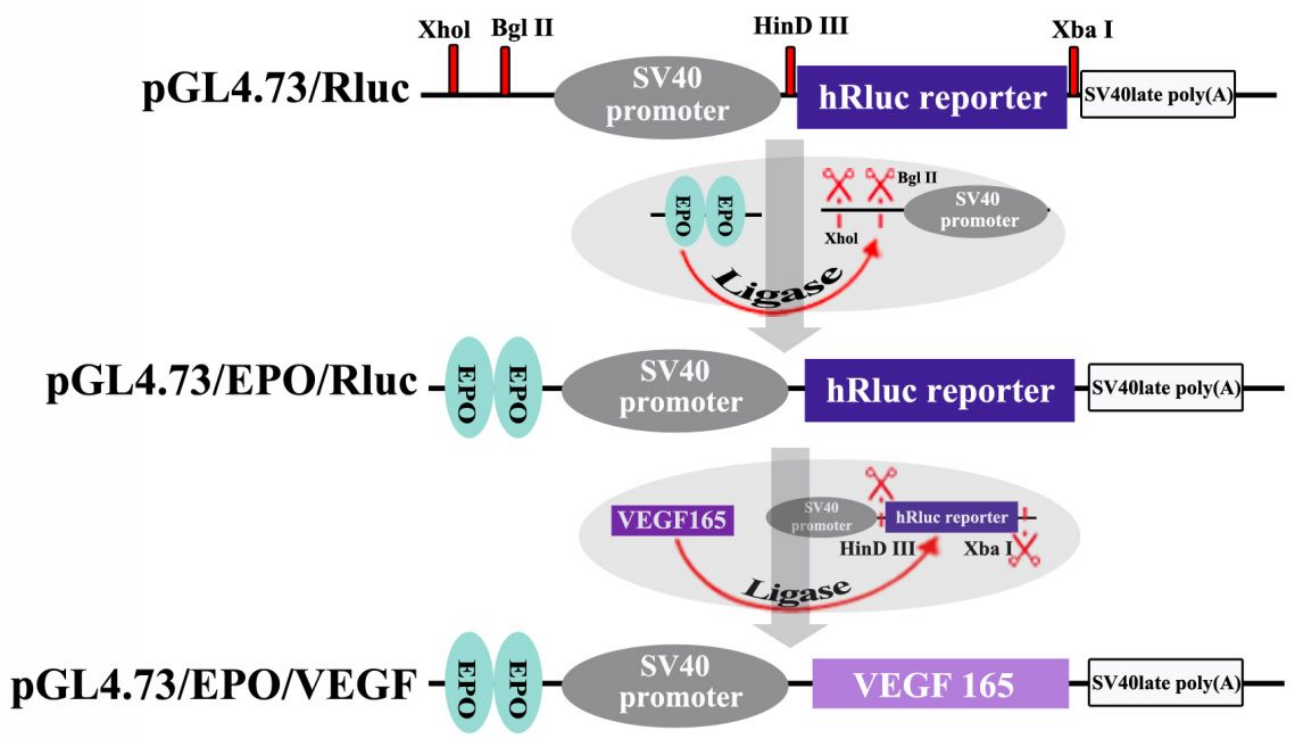

Figure S1 construction of circular DNA system with oxygen-sensing capacity. EPO indicates the erythropoietin enhancer which was the key element in biological oxygen sensing. SV40 was a promoter that was widely active in mammalian cells. 
A.

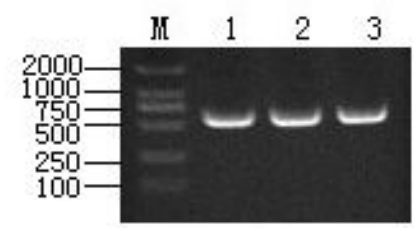

B.

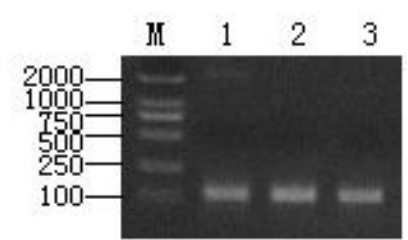

Figure S2 Verification of VEGF165 and tandem EPO enhancer gene fragments. A, Agarose Gel Electrophoresis demonstrated the presence of DNA products at the region between 500-600bp marker, corresponding to the size of VEGF165. 1, 2 and 3 indicated PCR products from templates pcDNA3 .1, pGL4.7/VEGF and pGL4.7/EPO/VEGF, respectively; B, Agarose Gel Electrophoresis demonstrated the presence of DNA products at the region about 100bp marker, corresponding to the size of two tandem EPO enhancers.1, 2 and 3 indicated PCR products from the templates EPO planmid, pGL4.7/EPO/Rluc and pGL4.7/EPO/VEGF, respectively.

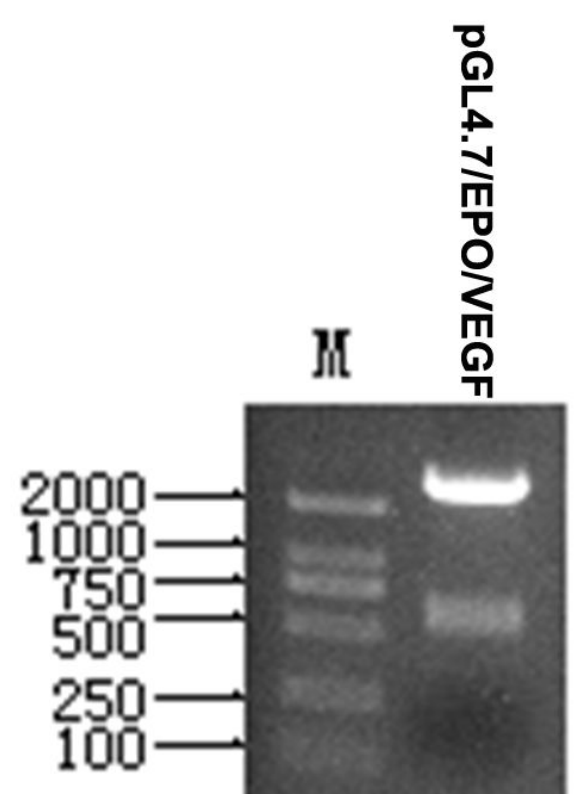

Figure S3. Verification of the successful insertion of VEGF165 in circular pGL4.7/EPO/VEGF. The circular DNA was digested by restriction endonuclease HindIII and XbaI. Two DNA fragments were detected. DNA fragment at the region of 500-750bp corresponded to the size of VEGF165 gene(576bp), while DNA fragment larger than 2000bp corresponded to the size of pGL4.7. 

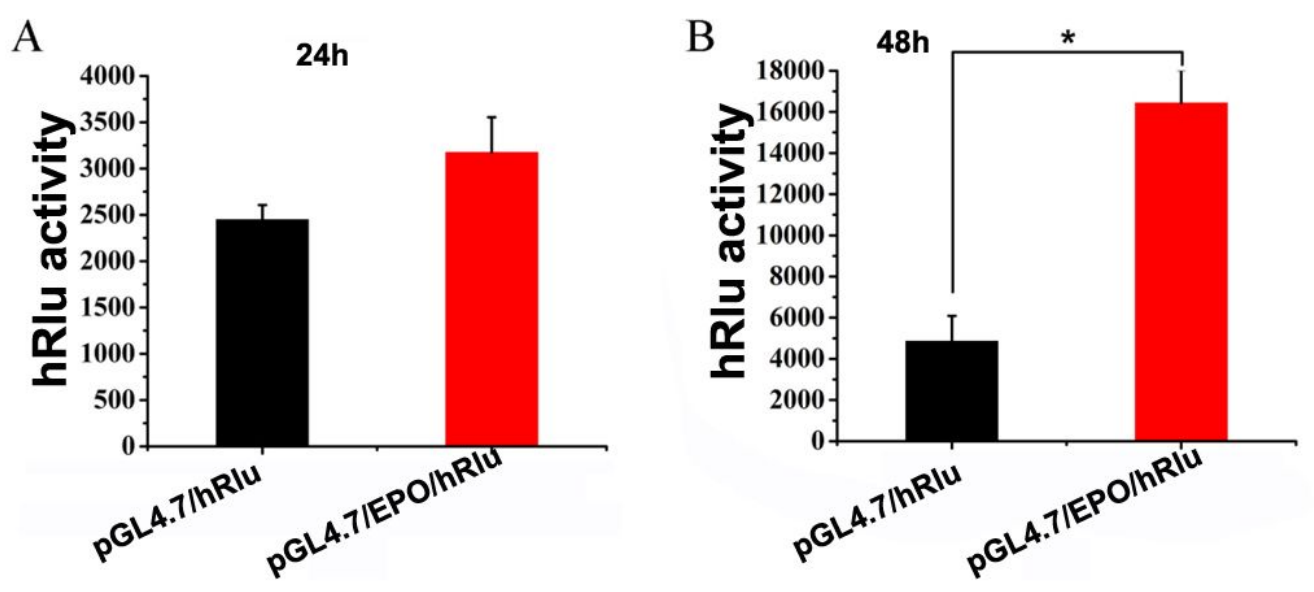

Figure S4. Oxygen sensing capacities of recombinant circular DNA under $\mathrm{CoCl}_{2}$ induced hypoxia. A, Under normoxia, no significant difference of reporter Rluc was detected between pGL4.7/Rluc and pGL4.7/EPO/Rluc; B, Under $\mathrm{CoCl}_{2}$ induced hypoxa, Rluc activity in pGL4.7/EPO/Rluc was significantly upregulated, about 3-4 folds of that in pGL4.7/Rluc. $* \mathrm{P}<0.001$
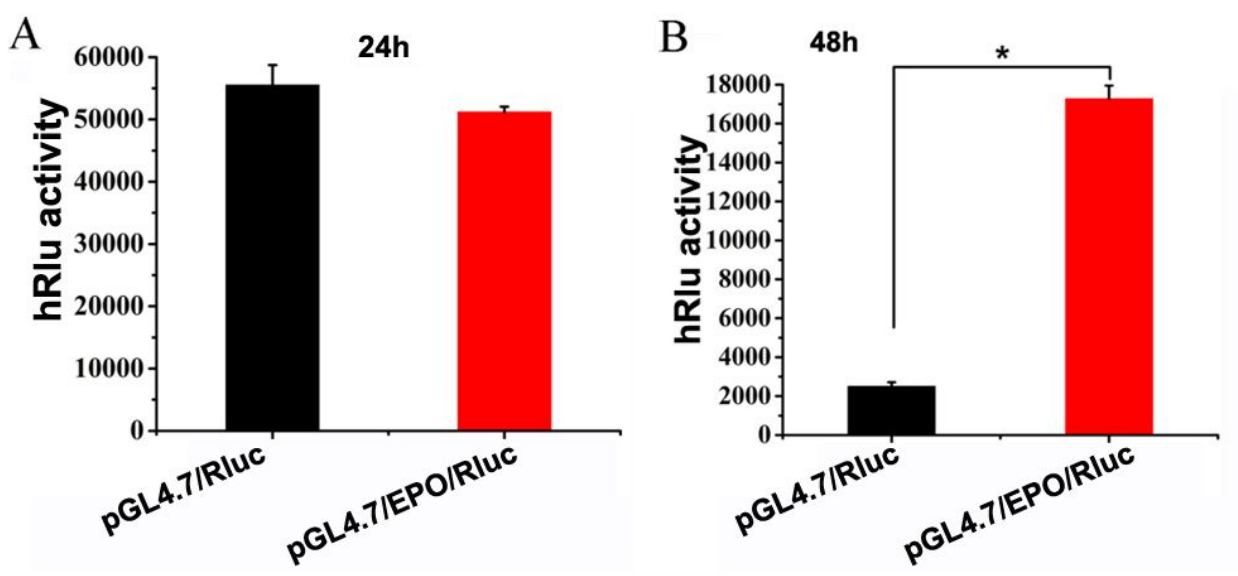

Figure S5. Oxygen sensing capacities of recombinant circular DNA in hypoxic incubator. A, Under normoxia, no significant difference of reporter Rluc was detected between pGL4.7/Rluc and pGL4.7/EPO/Rluc; B, In hypoxic incubator, Rluc activity in pGL4.7/EPO/Rluc was significantly upregulated, about 7-8 folds of that in pGL4.7/Rluc. $* P<0.001$ 


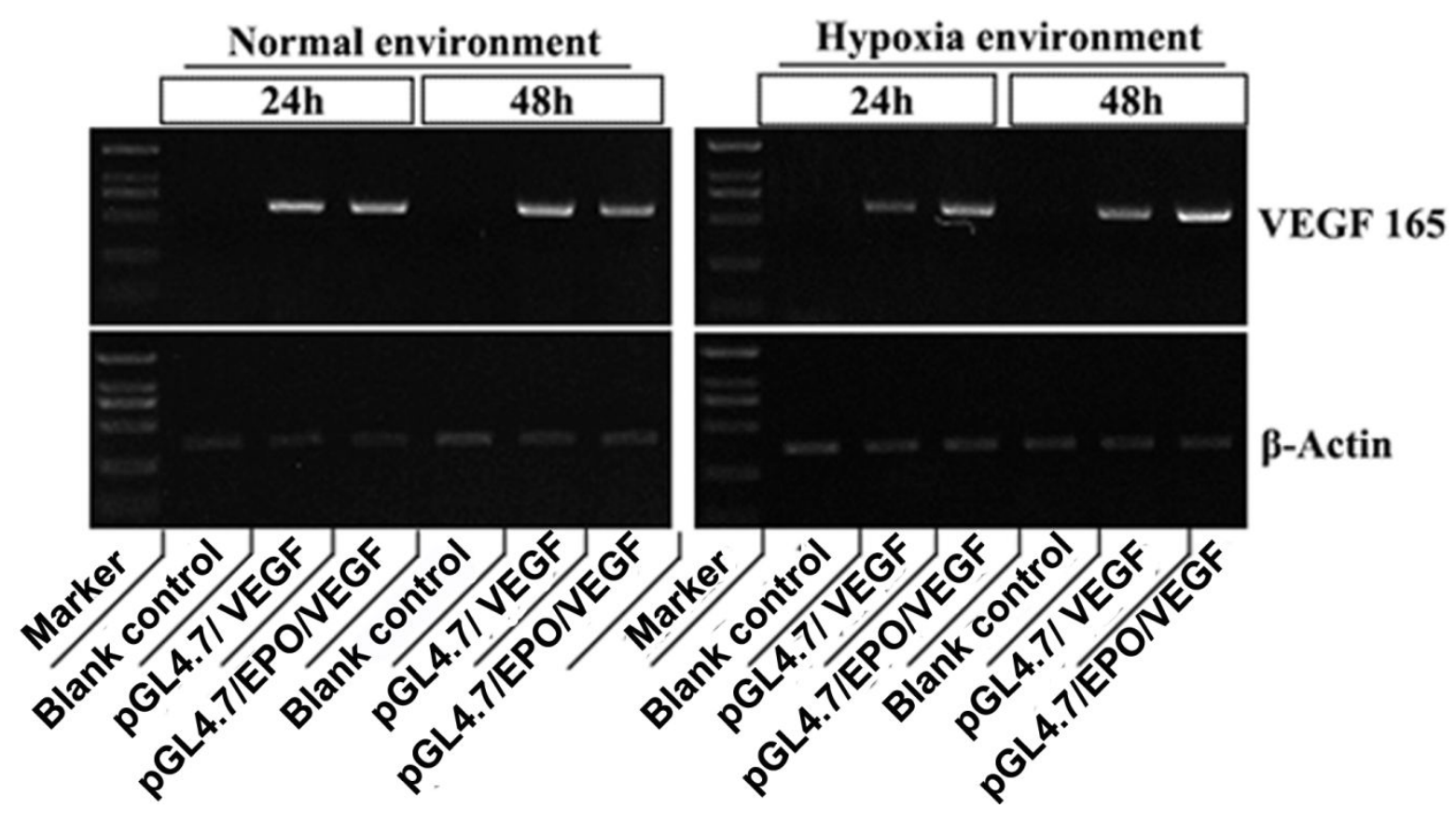

Figure S6. Oxygen sensing capacities of recombinant pGL4.7/EPO/VEGF system. Under normoxia, no difference of VEGF165 expression was detected between pGL4.7/ VEGF and pGL4.7/EPO/VEGF systems. However, under hypoxia, VEGF165 was significantly upregulated in pGL4.7/EPO/VEGF but in pGL4.7/ VEGF.

a
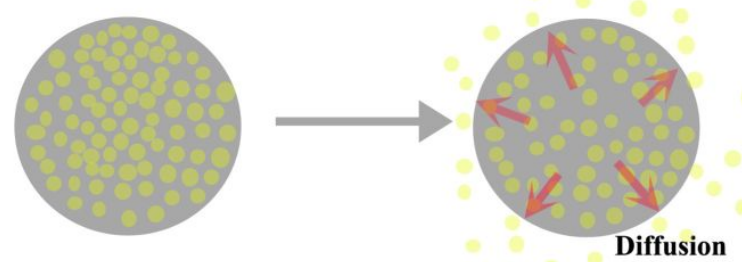

b
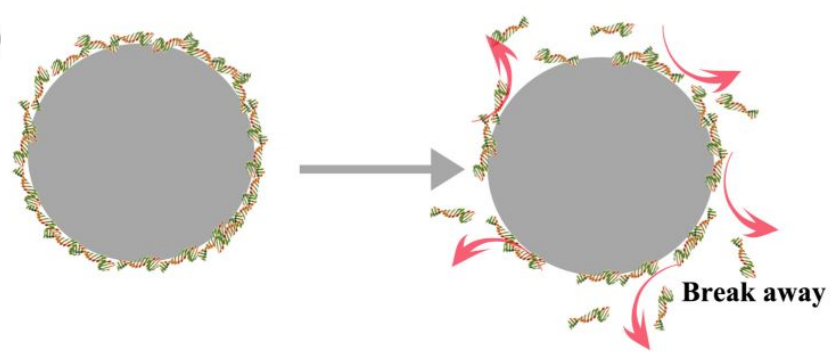

C
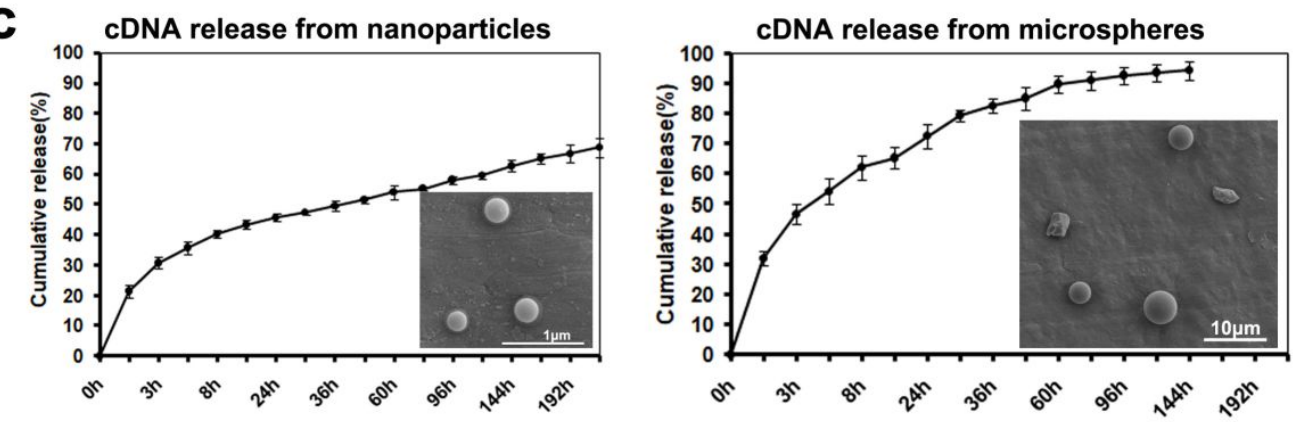
Figure S7. Schematic illustrating the release mechanisms of encapsulated melatonin within particles and adsorbed DNA on the surface of particles. a, encapsulated melatonin was released mainly via diffusion, the speed of which was negatively correlated with the size of particles(the smaller of the particles, the larger of the specific surface area, and the more rapid of drug release); $b$, adsorbed DNA on particles was released mainly via breaking away from electrostatic interactions, the speed of which was positively correlated with the size of particles(the larger of the particles, the farther between the particle core and the DNA, the easier for DNA to break away); c, cDNA release from nanoparticles and microspheres.

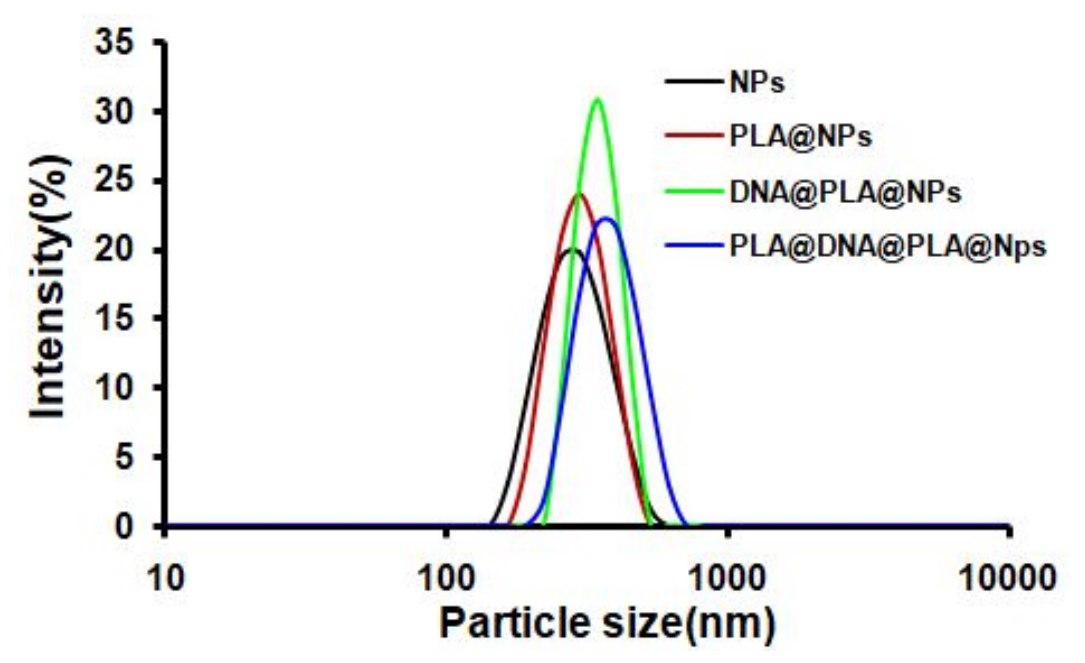

Figure S8. DLS demonstrated the sizes of particles increased with the LbL assemble. 


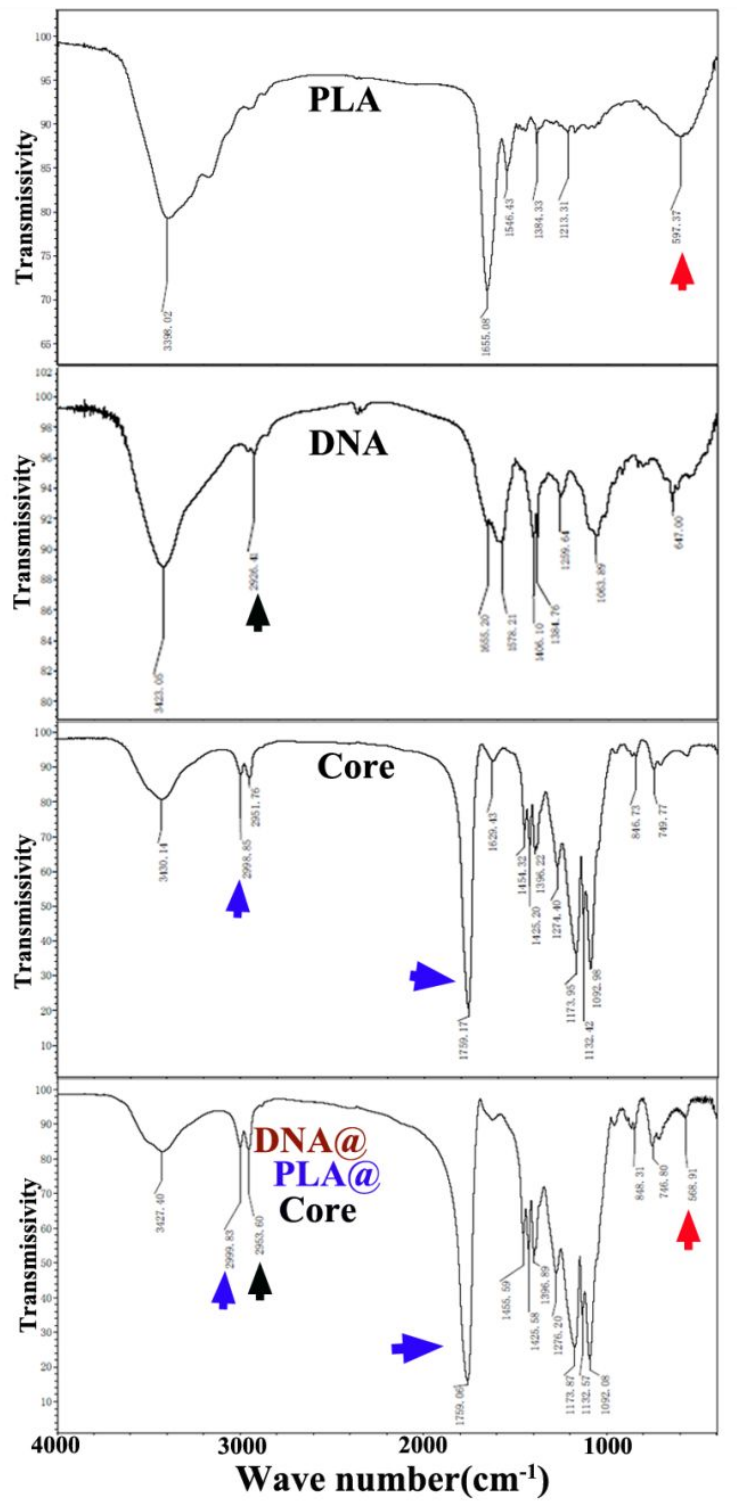

Figure S9. Near infrared diffraction analysis confirmed the accumulation of each component on the PLGA cores.

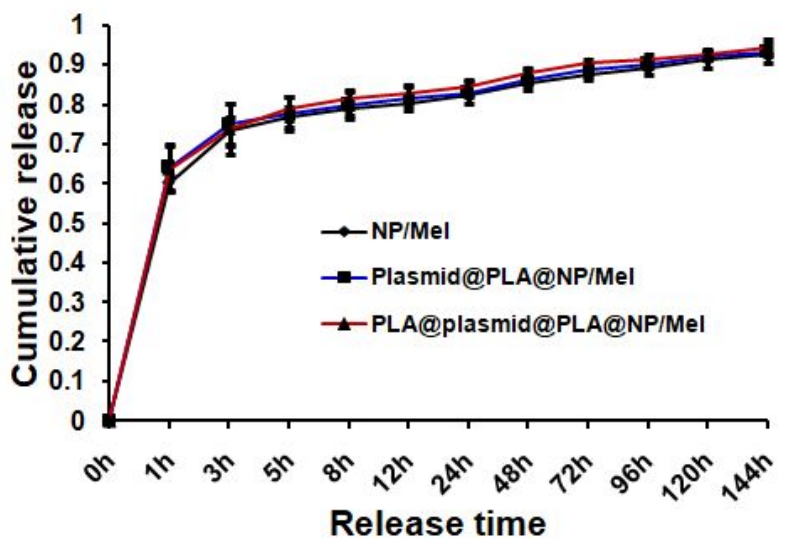

Figure S10. The release cures of melatonin from nanoparticles with different layers $(\mathbf{n}=3)$. 


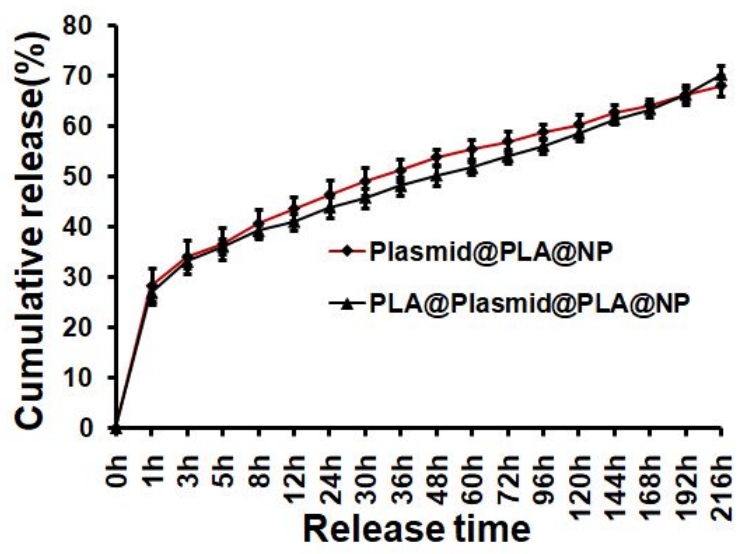

Figure S11. The release cures of circular DNA from nanoparticles with different layers $(\mathbf{n}=3)$.
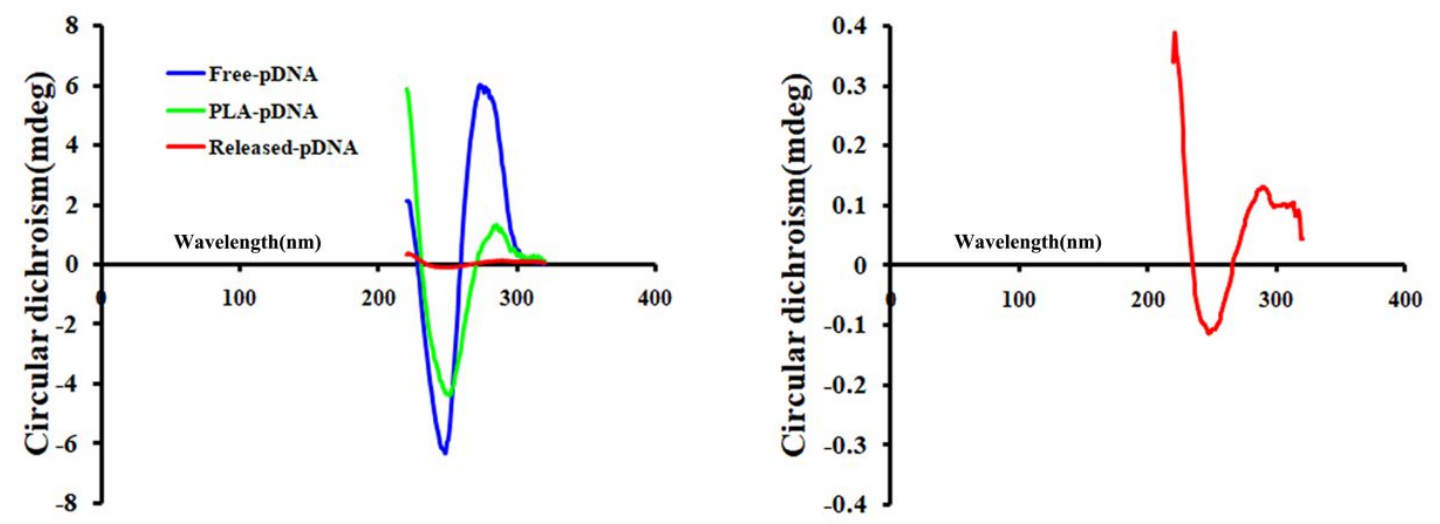

Figure S12. Circular dichroism spectra demonstrated the integrity of released DNA from MTSNP, and the released DNA was in form of PLA-DNA complex.

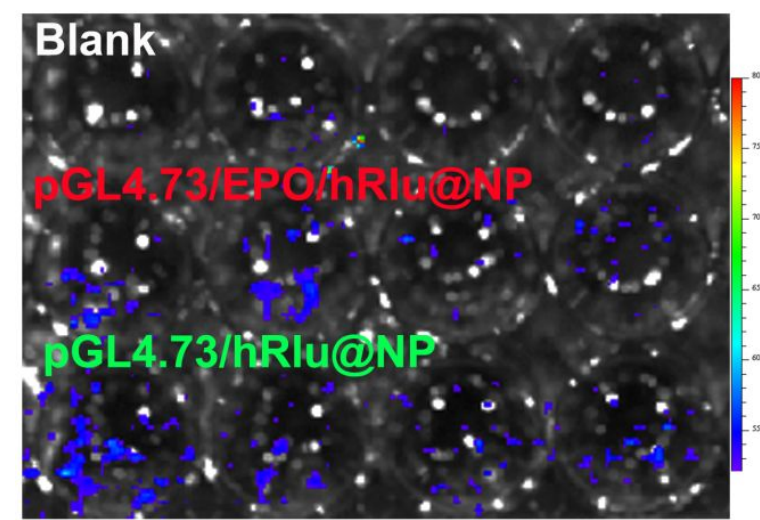

Normaxia

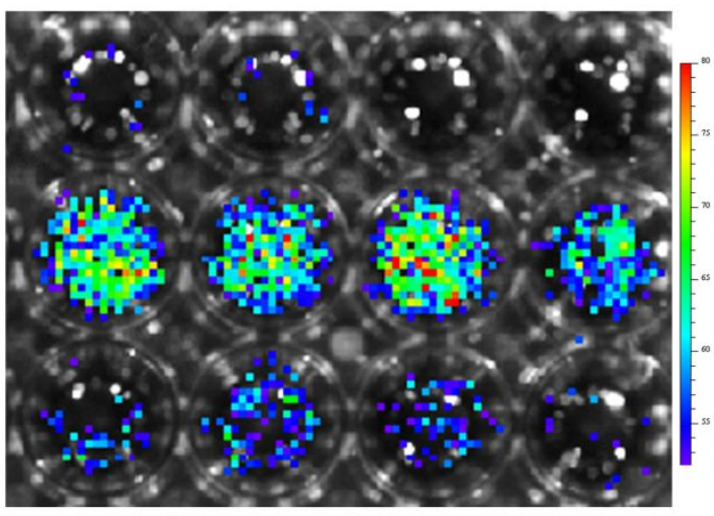

Hypoxia

Figure S13. Bioluminescent imaging of Rluc activities in host cells treated by MTSNP. 

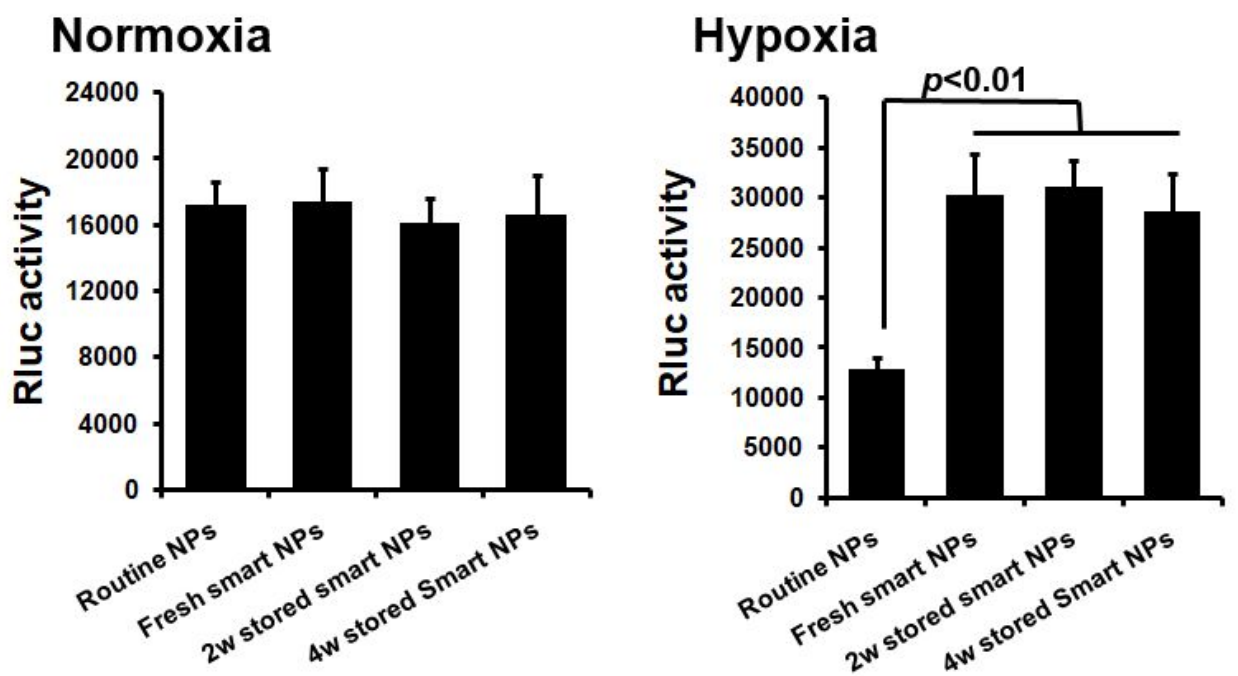

Figure S14. The biostability of smart NPs. Freshly prepared smart NPs and lyophilized smart NPs (stored at $4^{\circ} \mathrm{C}$ for $2 \mathrm{w}$ and $4 \mathrm{w}$ ) were incubated with $293 \mathrm{~T}$ cells for $48 \mathrm{~h}$ under normoxia and hypoxia respectively. Routine NPs were used as control. The activities of reporter gene Rluc in smart gene system were comparable among fresh smart NPs, $2 \mathrm{w}$-stored smart NPs and $4 \mathrm{w}$-stored smart NPs. .

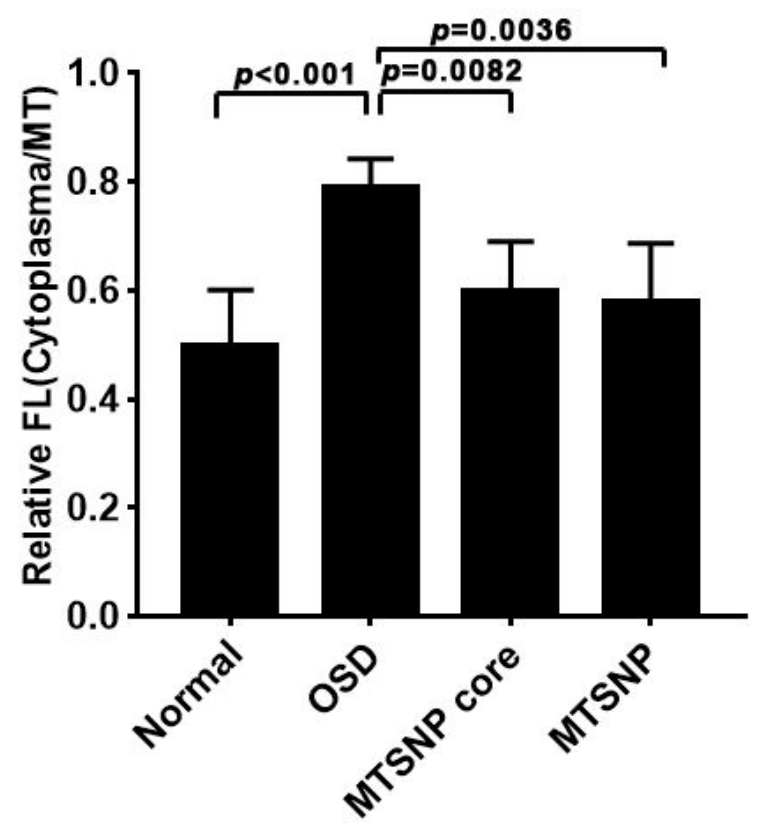

Figure S15. Quantitative analysis of the relative fluorescence of anti- cytochrome $\mathbf{C}$ staining in cytoplasm to that in mitochondria (FL: fluorescence). 


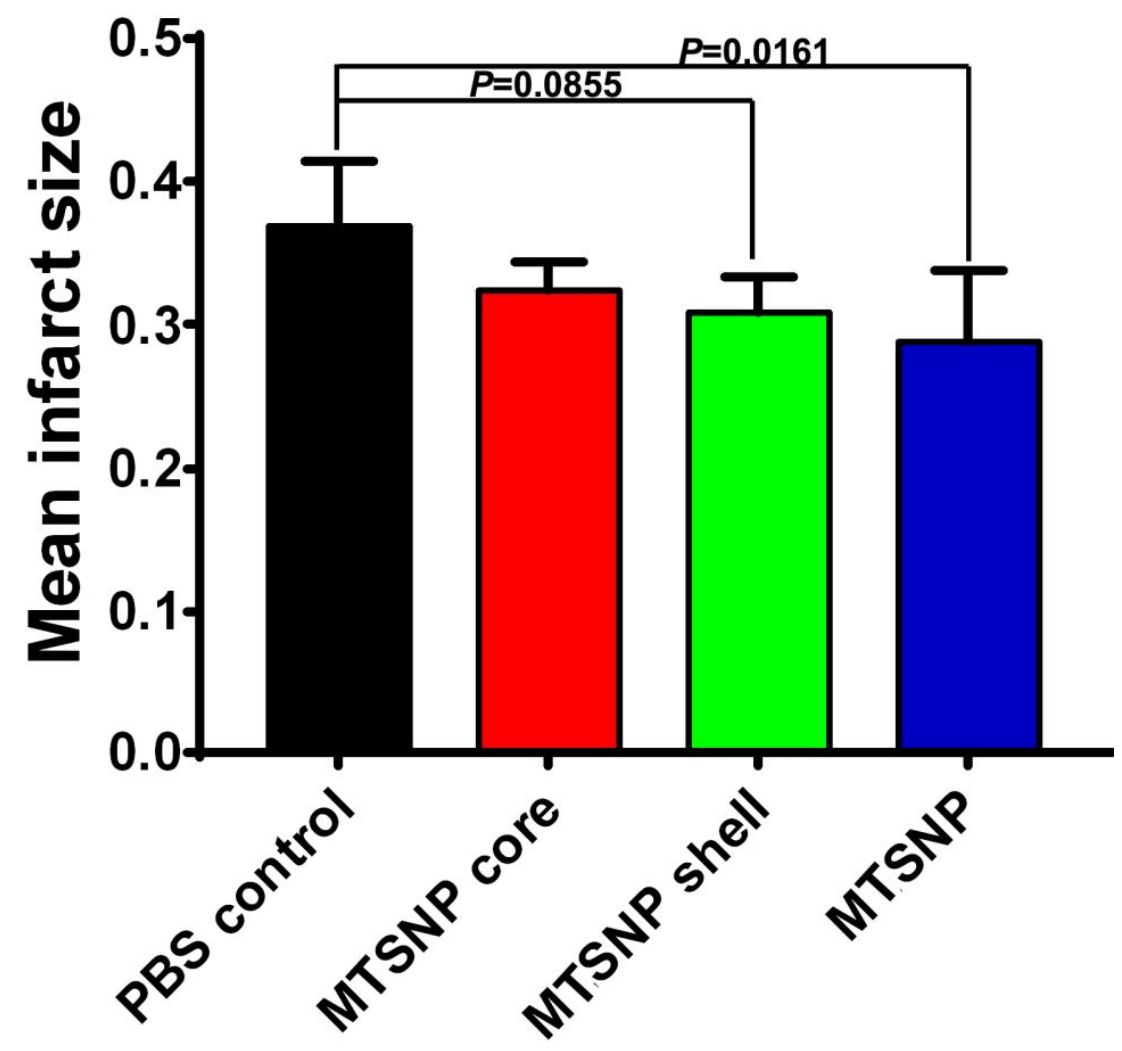

Figure S16. The mean infarct size. Mean infarct size $=$ (infarct outer perimeter + infarct inner perimeter)/(heart perimeter $+\mathrm{LV}$ inner perimeter). 


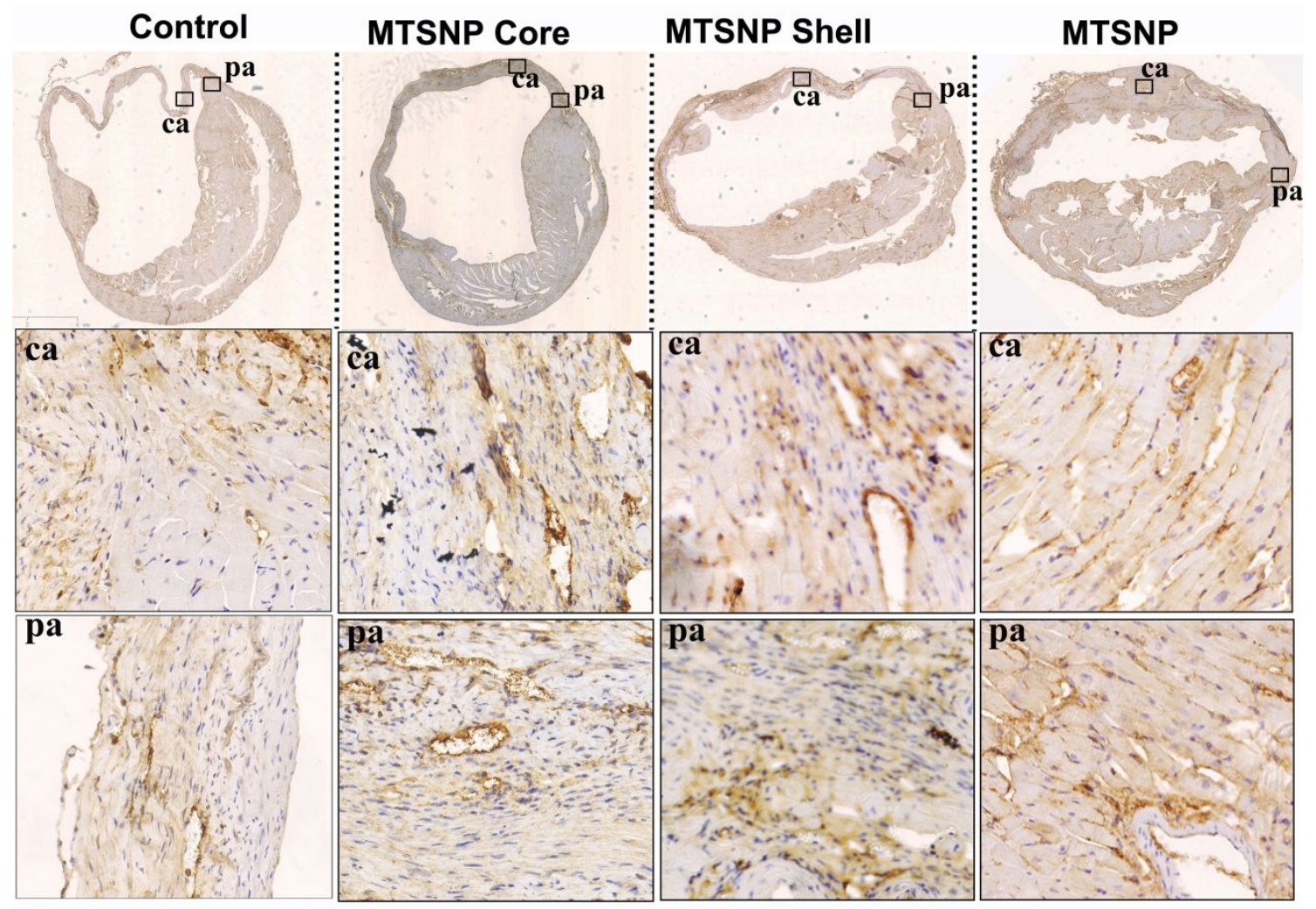

Figure S17. Immunostained heart sections against anti-vWF antibodies. Ca indicated infarct central area; pa indicated infarct peripheral area. 


\section{Reference}

1. Liu, Z.; Wang, H.; Wang, Y.; Lin, Q.; Yao, A.; Cao, F.; Li, D.; Zhou, J.; Duan, C.; Du, Z.; Wang, Y.; Wang, C. The Influence of Chitosan Hydrogel on Stem Cell Engraftment, Survival and Homing in the Ischemic Myocardial Microenvironment. Biomaterials. 2012, 33, 3093-3106.

2. Nie, J. J.; Qiao, B.; Duan, S.; Xu, C.; Chen, B.; Hao, W.; Yu, B.; Li, Y.; Du, J.; Xu, F. J. Unlockable Nanocomplexes with Self-Accelerating Nucleic Acid Release for Effective Staged Gene Therapy of Cardiovascular Diseases. Adv. Mater. 2018, 30, 1801570

3. Hosomi, S.; Watabe, T.; Mori, Y.; Koyama, Y.; Adachi, S.; Hoshi, N.; Ohnishi, M.; Ogura, H.; Yoshioka, Y.; Hatazawa, J.; Yamashita, T.; Shimazu, T. Inflammatory Projections after Focal Brain Injury Trigger Neuronal Network Disruption: An ${ }^{18}$ F-DPA714 PET Study in Mice. NeuroImage: Clinical 2018, 20, 946-954 